\title{
Simulation of an absorption chiller based on a physical model
}

\author{
Christian Fleßner Stefan Petersen Felix Ziegler \\ Technische Universität Berlin, Fachgebiet Maschinen- und Energieanlagentechnik, \\ Marchstr. 1810587 Berlin
}

\begin{abstract}
Previous works on simulation of air conditioning systems with absorption chillers in conjunction with detailed experimental analysis have shown a need for a more detailed and generalized modelling and simulation of heat and mass transfer processes in absorption chillers. An existing model for absorption is adapted to be applicable for subcooled or superheated liquids and for the desorption process. New classes compatible with the Modelica_Fluid library (beta 2) for these sub-processes are developed. A media model for evaporating aqueous salt solutions based on Modelica.Media is developed and implemented accordingly. Subsequently, simulations of a complete absorption chiller are conducted and compared with experimental data. The comparison of simulations under stationary conditions show a good agreement with experimental data while the transient behaviour of the plant is not yet fully implemented in the model.

Keywords: heat and mass transfer; falling film; aqueous salt solutions; Modelica.Media; Modelica_Fluid
\end{abstract}

\section{Introduction}

Absorption chillers are an advantageous option for reduction of primary energy demand for airconditioning. The necessary heat can be provided by solar thermal collectors for example. To increase the market share of these cooling systems, more efficient and more compact systems with low driving temperature requirements are necessary.

Previous works on building system simulations with absorption chillers [1] have used simple linear models for absorption chillers based on empirical coefficients. For newly designed systems or systems yet to be designed these parameters are often not readily available. Also, the results of a detailed experimental analysis show significant deviations from the simple assumptions made during design [2]. Physical simulation enables a more reliable design process and improvement of absorption chillers with reduced experimental effort. The models used for the simulation must be as generalized as possible to be able to vary parameters without the need for preliminary experiments. To reach this, models based on fundamental physical properties are a favourable option. On the other hand the complexity of the model must not exceed certain limits to enable the simulation of complete systems within acceptable time limits.

In the current work a thoroughly examined sorption chiller is modelled to enable an evaluation of model quality for a complete system. The focus of modelling is on the absorption and desorption process since these are critical for overall process efficiency.

The investigated system is a compact absorption chiller with a nominal refrigerating capacity of $10 \mathrm{~kW}$ and the working pair water/lithium bromide. All heat exchangers are built as falling film units with the external media passing through horizontal tubes in countercross-flow to the internal (process side) falling film, flowing on the outside of the tubes. Detailed experimental data and the complete specification of this chiller is presented in [2].

The simulation tool used in this work is Dymola 6.1 with version 2.2.1 of the Modelica standard library and the beta 2 Version of the Modelica_Fluid library.

\section{Model library}

A new library for the simulation of absorption chillers was developed. The library is based on and is compatible with the Modelica_Fluid beta 2. It consists of some modified components of Modelica_Fluid, extended media models based on Modelica.Media and newly developed models for absorption and desorption or evaporation and condensation respectively in falling film flow as the mostly used unit operation in absorp- 
tion chillers. The most significant adapted models were the integration of heat transfer models with variable heat transfer coefficients with the the DistributedPipe model.

Modelling concentrates on the thermodynamic modelling of heat and mass transfer in falling film flow. The pressure losses in the steam phase and the falling film are considered to be negligibly small. Therefore hydrodynamical aspects generally are considered on a significantly simplified level.

Some parts of the absorption chiller including the sumps of the falling film heat exchangers, the pumps and much of the external piping are not considered in this model. The neglect of these parts is expected to lead to inaccuracies of the model's dynamic behaviour. Control of the chiller was not considered as well since the current work is mainly concerned with the modelling and simulation of the internal process.

\subsection{Media model}

For the Media model a new partial model PartialMixtureTwoPhaseMedium was derived from the Modelica.Media partial models PartialTwoPhaseMedium and PartialMixtureMedium to allow for evaporating mixtures analogously to the model developed for aqueous sodium chloride solution in [3]. The Correlations for density, specific enthalpy, specific isobaric heat capacity and specific entropy are taken from [4], while heat conductance and dynamic viscosity are computed according to Lee et. al [5]. The final model for the aqueous lithium bromide solution assumes pure water in the steam phase since the salt has a negligible vapour pressure within the valid range of the medium model. This allows to refer to the Modelica.Media.Water models for this part. The final model is not well suited for full two phase flow simulation since it is explicit in pressure, temperature and mass fraction which does not allow for a proper description of the two phase dome. For the scope of this study the possibility of surface evaporation is sufficient as it is reasonable to assume pure vapour without liquid droplets in all units. In the following parts only the newly developed models are described since most adaptations of existing models includes only minor modifications.

\subsection{Film model}

The model for absorption and desorption is derived from an existing model [6]. The full derivation of this model is to be found there. In the current work only the basic ideas and the main equations can be shown. Both the original model and the further development shown here assume that absorption and desorption only take place during the falling film mode along the horizontal tube with no mass transfer occurring in the droplet formation and falling droplet modes. Furthermore ideal mixing during droplet formation is assumed allowing for simple connections between single tubes. The geometry of the film is simplified to a straight onedimensional falling film.

The calculation of heat transfer coefficients in the model is based on the stagnant film theory of Nußelt resulting in eq. (1)

$$
\alpha_{f i l m}=\frac{\lambda}{\delta}=\sqrt[3]{\frac{\lambda^{3} g \rho_{s}^{2}}{3 \dot{\Gamma} \eta}}
$$

with the mass flow rate per one side tube length $\dot{\Gamma}$, the thermal conductance of the solution $\lambda$, the dynamic viscosity $\eta$ and the density of the solution $\rho$.

By means of a coordinate transformation from the running length $z$ to a running time $t_{r}$, the mass transfer can be regarded as instationary diffusion into a semiinfinite body since the concentration in the bulk phase is constant along the falling film on each horizontal pipe. A uniform entrance concentration can be derived form the assumption of ideal mixing during droplet formation. This results in eq. (2) to describe the concentration profile along the film thickness.

$$
\frac{c_{\mathrm{H}_{2} \mathrm{O}}(y, t)-c_{\mathrm{H}_{2} \mathrm{O}}(y, t=0)}{c_{\mathrm{H}_{2} \mathrm{O}}(y=0, t)-c_{\mathrm{H}_{2} \mathrm{O}}(y, t=0)}=\operatorname{erfc}\left(\frac{y}{2 \sqrt{D t_{r}}}\right)
$$

The average mass transfer coefficient for a single tube is defined by eq. (3)

$$
\beta=\frac{m_{a b s}}{\bar{\tau} A\left(x_{L i B r}^{\prime}-x_{L i B r, i}\right)},
$$

with the overall absorbed mass for a single tube $m_{a b s}$, the mean time of exposure $\bar{\tau}$ and the mass fractions $x$. The local mass flow rate into the film is described with Fick's first law. The Introduction of eq. 2) into Fick's law along with the assumption that the density at the interface $\rho_{i}$ and the density on entry can both be approximated with an average density $\bar{\rho}$ allows the integration of the mass flow rate over the time of exposure. This leads to the calculation of the mass transfer coefficient with eq. (4)

$$
\beta=\frac{2}{\sqrt{\pi}} \cdot \bar{\rho} \cdot \sqrt{\frac{D}{\bar{\tau}}} .
$$

The original model assumes a stationary temperature profile and a latent heat that is large compared 
to the sensible heat. Therefore only the heat of absorption has to be transferred from the interface to the cooling water which determines the coupling of heat and mass transfer as given by eq. (5)

$$
\frac{m_{a b s}}{\bar{\tau}} \Delta h_{a b s}=A \cdot \alpha_{f i l m} \cdot\left(T_{i}-T_{w}\right) .
$$

The interface temperature $T_{i}$ and the wall temperature $T_{w}$ are to be assumed as constant. The interface mass fraction and temperature are connected by the equilibrium relation $T_{i}=T_{\text {sat }}\left(x_{\mathrm{H}_{2}} \mathrm{O}(y=0, t), p\right)$ with the constant pressure $p$.

This model is extended to be applicable to subcooled and superheated conditions in the bulk liquid phase of the film. To reach this the film is divided into two layers, where the heat transfer from the film surface to the core of the film is coupled with mass transfer from or to the vapour phase while the heat transfer in the inner layer between the film's core and the pipe wall is assumed to be independent from mass transfer on the surface. Heat transport in both layers is calculated separately according to Nußelt's theory and are coupled with an interface temperature between the layers. As a first approximation this temperature $T_{S}$ is constantly set to be the mixed cup temperature $T^{\prime \prime}$ at the outlet derived from the energy balance. In this model the the distribution of the film thickness between the layers is an arbitrary constant that has to be fitted according to experimental data. A similar assumption was already made by Jeong and Garimella [7] for a laminar falling film. They assumed a linear temperature profile with a sharp increase in temperature because the heat of absorption is released locally at the surface therefore inducing a steeper gradient. In contrast to the still greatly simplified model detailed here they considered a variable depth of the bending profile. Furthermore they did neither make the simplified assumption of a constant temperature profile over the complete tube nor did they assume the surface concentration as constant. This leads to an extremely complex model requiring a discretisation of a single tube into 100 elements, making the model unsuitable for the simulation of a complete chiller. In the current work the thickness of the outer layer is set to be $10 \%$ of the overall film thickness. Jeong and Garimella [7] have calculated a similar distribution for a flow angle of $90^{\circ}$.

The schematic of a single absorber tube with its concentration and temperature profiles is shown in Figure 1 with the sharper increase of temperature towards the phase interface highlighted. The desorption process is described in the same way, leading to inverse profiles.

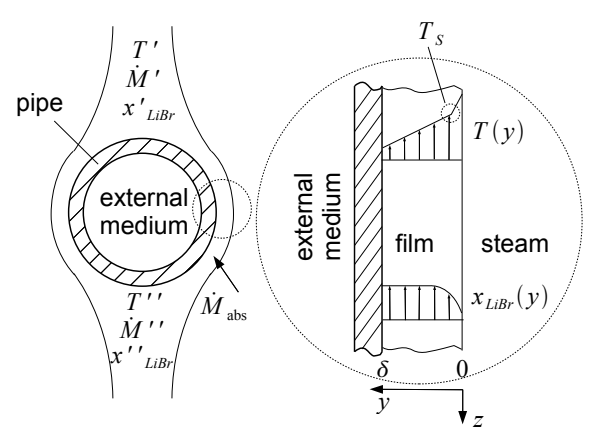

Figure 1: Schematic of a single absorber tube, concentration and temperature profile

The average absorbed or desorbed mass flow rate for a single tube $\dot{M}_{a b s}=m_{a b s} / \bar{\tau}$ is calculated with eq. (6)

$$
\dot{M}_{a b s}=\beta \cdot A \cdot\left(x_{L i B r}^{\prime}-x_{L i B r, P h}\right) .
$$

The heat flow transferred to or from the tube wall and from there to the cooling water is given by eq. (7)

$$
\dot{Q}_{\text {cool }}=A \cdot \alpha_{\text {film,inner }} \cdot\left(T_{S}-T_{w}\right) .
$$

In the model presented here the coupling between heat and mass transfer is set up slightly different to eq. (5) since it is assumed that coupling between heat and mass transfer occurs only in the upper layer which is directly influenced by the heat of absorption resulting in eq. (8)

$$
\frac{m_{a b s}}{\bar{\tau}} \Delta h_{a b s}=\alpha_{f i l m, u p p e r} \cdot A \cdot\left(T_{i}-T_{S}\right),
$$

with the relevant heat transfer occurring between the interface temperature $T_{i}$ and the Temperature at the bend of the temperature profile $T_{S}$.

No mass storage in the liquid phase is assumed. Therefore the overall mass balance is defined in eq. (11)

$$
0=\dot{M}^{\prime}+\dot{M}^{\prime \prime}+\dot{M}_{a b s} .
$$

Accordingly the component mass balance of lithium bromide is given as eq. (10)

$$
0=x_{L i B r}^{\prime} \cdot \dot{M}^{\prime}+x_{L i B r}^{\prime \prime} \cdot \dot{M}^{\prime \prime} .
$$

The energy balance eq. (11) is instationary to allow for heat storage in the balance volume.

$$
\begin{aligned}
\frac{\mathrm{d} U}{\mathrm{~d} t=} & h_{L}\left(T^{\prime}, x^{\prime}\right) \cdot \dot{M}^{\prime}+h_{L}\left(T^{\prime \prime}, x^{\prime \prime}\right) \cdot \dot{M}^{\prime \prime} \\
& +h_{v, s a t}(p) \cdot \dot{M}_{a b s}+\dot{Q}_{c o o l}
\end{aligned}
$$

The vapour phase is considered to consist entirely of saturated pure water steam. Mass transfer resistance in the vapour phase and on the phase boundary 
is neglected. Thermal equilibrium on the interface is assumed for the liquid phase. The mathematical representation of the Generator in the current model is completely identical to that of the absorber and is therefore not shown here.

Evaporator and condenser are completely described with Nußelt's stagnant film theory. No mass transfer resistances are accounted for. Equilibrium is assumed in both the vapour and the liquid phase. The heat transfer and balance equations are identical to absorber and generator. No composition has to be considered since pure water is used as cooling agent.

The implementation in Modelica includes partial models for film heat and mass transfer and final models based on the equations above. These models are selectable from separate models for falling film flow including the balance equations and the coupling between heat and mass transfer. Flow reversal is implemented for the vapour phase connectors to allow the usage of the film models for absorption/desorption and evaporation/condensation respectively.

\subsection{System models}

The models for the falling film are coupled with the modified tube models via HeatPort connectors from Modelica.Thermal as shown in Figure 2. These pipe segments are then connected with FluidPorts according to each units flow path. A more flexible solution allowing for selection of different flow paths and number of passes is desirable but is not implemented yet.

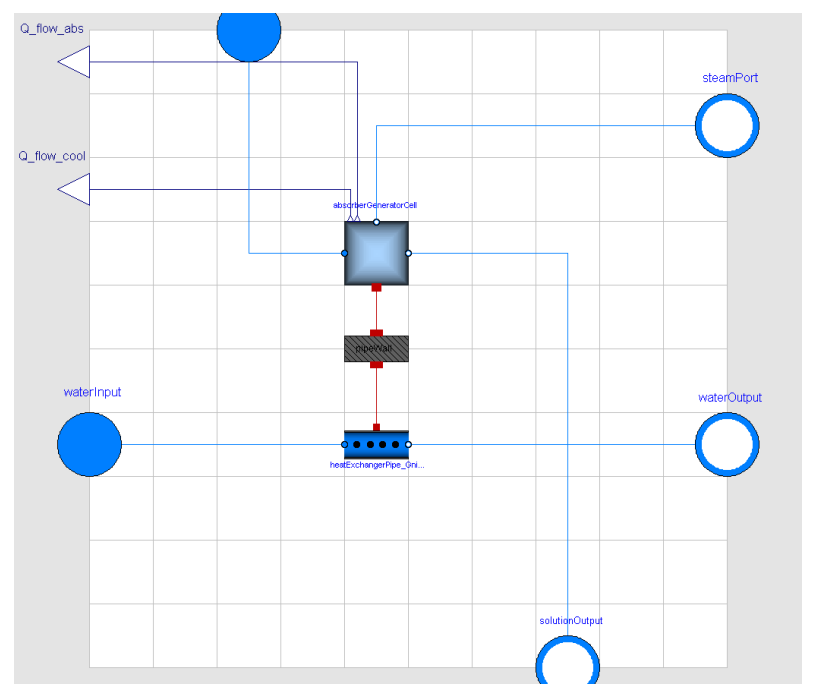

Figure 2: Diagramm of AbsorberGeneratorPipe

Each vapour phase is represented by a MixingVolume which is connected to the heat exchangers by Flu-
idPorts. Constant mass flow rates are prescribed for internal and external circuits. The temperatures of the external flows are kept constant, while the entry temperatures and concentrations of the internal solution circuit and the entry temperature of the evaporator circulation are fed back from the respective output values. The representation of the overall model in Dymola is shown in Figure 3.

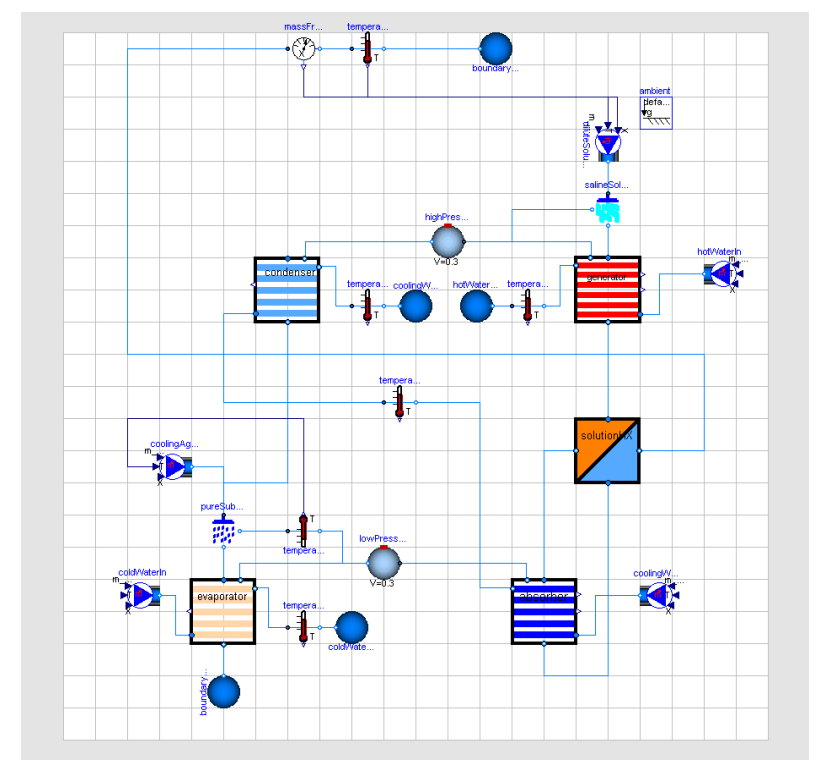

Figure 3: Diagramm of complete model

\section{Comparison with experimental re- sults}

In most cases the simulation is conducted with constant external conditions. From a cold start the whole system becomes fully stationary after less than $100 \mathrm{~s}$ simulated time. This relatively fast reaction is reasonable as some major points of heat and mass storage in the plant (heat exchanger sumps an external piping for instance) are not included in the model and mass storage in the falling film is generally neglected. Simulations with ideal steps in one of the three external temperature levels have been conducted showing a qualitatively correct response. Figure 4 exemplary shows the response of the model to an instantaneous increase in driving temperature with all other external conditions left constant. The response is qualitatively plausible with all final values identical to the stationary simulations. Due to the reasons mentioned above the simulated delay is much shorter than the delay observed in the real plant, where [8] reports measured delays 10 times as high as in the current simulations. 


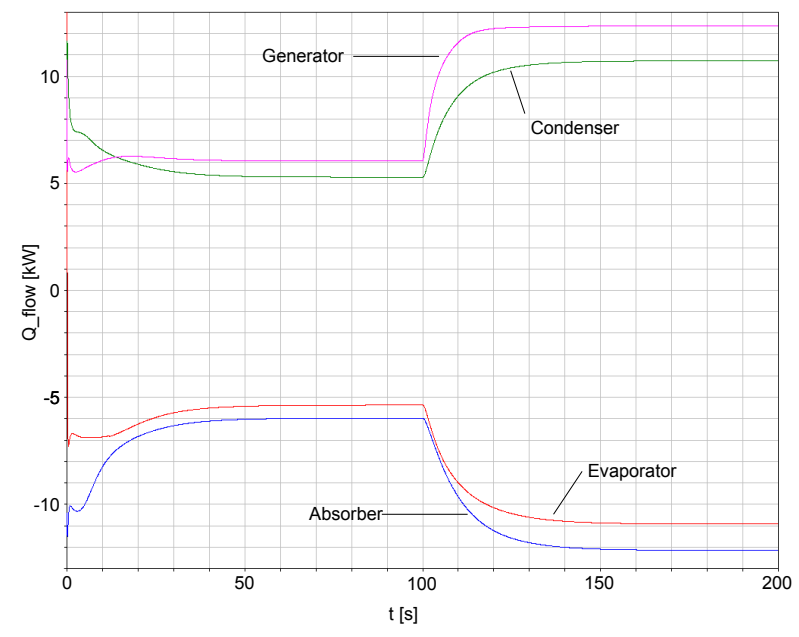

Figure 4: Response of external heat flows to step in driving temperature $\left(55^{\circ} \mathrm{C}\right.$ to $75^{\circ} \mathrm{C}$ at $\left.100 \mathrm{~s}\right)$

The simulation of the complete systems yields stationary heat fluxes which show a positive deviation of $5 \%$ to $15 \%$ from experimental data. The deviation increases with the driving temperature. the results for nominal operating conditions (external conditions: $75^{\circ} \mathrm{C}$ generator inlet, $27^{\circ} \mathrm{C}$ cooling water inlet, $18^{\circ} \mathrm{C}$ cold water inlet) are shown in Figure 5. One plausible reason for this systematic deviation is that incomplete wetting of the heat exchanger surfaces is not considered in the model. In [2] an average wetting between $80 \%$ and $90 \%$ with significant deviations between operating conditions was reported. A rough approximation where the internal heat exchanger surface was reduced by $20 \%$, while the external area was constant, led to significantly improved results for nominal conditions. The generator shows a larger deviation than the other units. This indicates that the generalisation of assumptions concerning heat and mass transfer as well as wetting for absorber and generator is not fully adequate. Nevertheless, the reults are generally in good agreemant with the experimental data.

The internal temperatures and concentrations in the falling film including superheating and subcooling that were measured in [2] could also be approximated in the simulation giving further credibility to the assumptions made in the model. Figure 6 shows the arithmetic average of the calculated and measured internal temperatures for better comparison.

\section{Conclusion}

Overall the accuracy of the stationary simulations with this simple model which is nonetheless not strongly

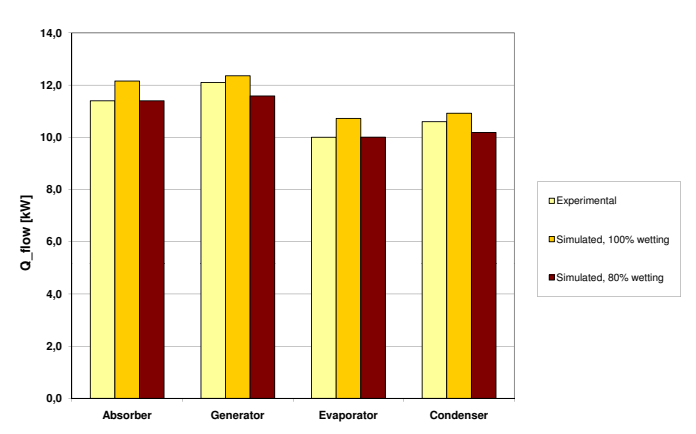

Figure 5: Stationary heat flow at nominal conditions

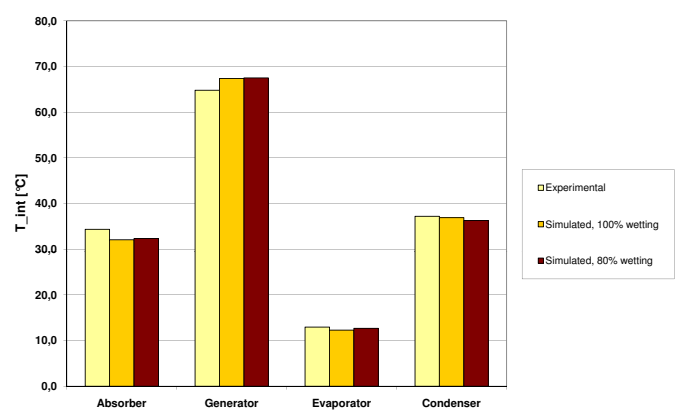

Figure 6: Stationary internal temperatures (arithmetic average of inlet and outlet) at nominal conditions

dependent on empirical fitting parameters is rather good. The comparison of the simulated results with experimental data shows good agreement in overall performance though an evaluation of the accuracy of the predicted local transfer coefficients has yet to be done by more detailed experiments. Pending issues include the yet incomplete modelling of the dynamic behaviour of the whole system and the long simulation times required for simulation of a complete chiller. Since the implementation of flow reversal and the structure of the media model generate large amounts of non-linear equations, a single simulation run over $100 \mathrm{~s}$ simulated time with constant external conditions takes more than an hour of simulation time. In its current state the model is more suitable for parameter generation for simpler models than for system simulation. Therefore further development will be conducted including the adaptation of the library to the Modelica_Fluid 1.0 Library to resolve current performance issues. The hydrodynamical modelling also needs some improvement as a sys- 
tematic and physically founded consideration of incomplete wetting seems to be worthwhile for generalisation of the model. Here care has to be taken to avoid an overly complex model making simulation of complete chillers unfeasible.

\section{References}

[1] Annett Kühn, José Luis Corrales Ciganda, Felix Ziegler. (2008): Comparison of control strategies of solar absorption chillers, Proceedings of the 1st International Conference on Solar Heating, Cooling and Buildings (Eurosun), 7-10 October 2008, Lisbon, Portugal

[2] Annett Kühn, Lukas Enke, Felix Ziegler (2008): Detailed Analysis of A $10 \mathrm{~kW} \mathrm{H}$ O/LIBR Absorption Chiller, International Sorption Heat Pump Conference 2008, 23-26 September 2008, Seoul

[3] Katja Poschlad, Manuel A. Pereira Remelhe, Martin Otter (2006): Modeling of an experimental Batch Plant with Modelica, Proceedings of the 5th International Modelica Conference 2006, Vienna

[4] Günther Feuerecker (1994): Entropieanalyse für Wärmepumpensysteme: Methoden und Stoffdaten, Ph.D.-Thesis Technische Universität München

[5] R.J. Lee, R.M. DiGuilio, S.M. Jeter, A.S. Teja (1990): Properties of Lithium Bromide-Water Solutions at High Temperatures and Concentrations - II Density and Viscosity in ASHRAE Transactions, Paper 3381, RP-527, pp. 709-714 Atlanta: American Society of Heating, Refrigerating and Air-Conditioning Engineers

[6] Hein Auracher, Arnold Wohlfeil, Felix Ziegler (2008): A simple physical model for steam absorption into a falling film of aqueous lithium bromide solution on a horizontal tube, Heat and Mass Transfer 44; 1529-1536

[7] Siyoung Jeong, Srinivas Garimella (2002): Falling-film and droplet mode heat and mass transfer in a horizontal tube $\mathrm{LiBr}$ /water absorber: International Journal of Heat and mass Transfer $45 ; 1445-1458$
[8] Paul Kohlenbach (2006): Solar Cooling with absorption chillers: Control strategies and transient chiller performance: Ph.D.-Thesis Technische Universität Berlin; DKV-Forschungsbericht Nr. 74 